
\title{
25 Research Soure \\ Ideal Stage of Embryo Transfer to Improve \\ Outcomes for Women of Advanced Maternal Age
}

\section{Xue Wang}

Peking Union Medical College Hospital

Yaling Xiao

Peking Union Medical College Hospital

Zhengyi Sun ( $\nabla$ sunzhengyi@263.net )

Peking Union Medical College Hospital https://orcid.org/0000-0001-6363-9723

Jingran Zhen

Peking Union Medical College Hospital

Qi Yu

Peking Union Medical College Hospital

\section{Research}

Keywords: assisted reproductive technology, clinical pregnancy, embryo transfer, in vitro fertilisation, live birth

Posted Date: September 2nd, 2021

DOI: https://doi.org/10.21203/rs.3.rs-847328/v1

License: (c) (i) This work is licensed under a Creative Commons Attribution 4.0 International License.

Read Full License 


\section{Abstract \\ Background}

The purpose of this retrospective study was to optimise the transplantation strategy for women of advanced maternal age to achieve live births within the shortest time.

\section{Methods}

Data were collected from patients older than 40 years who underwent assisted reproductive therapy at our centre from 1 January 2009 to 31 December 2019. A total of 1233 cases of fresh cleavage embryo transfer cycles, 280 cases of frozen-thawed blastocyst transfer cycles, and 26 cases of frozen-thawed cleavage embryo transfer cycles were included. Multivariable logistic regression was performed to adjust for confounding factors.

\section{Results}

The main outcome was the live birth rate. The secondary outcomes were the clinical pregnancy rate, spontaneous abortion rate, and neonatal outcomes. We found that the blastocyst formation rate of patients older than 40 years was $23.5 \%$, the freezing cycle rate was $19.8 \%$, and the fresh embryo transfer rate was $83.0 \%$.

\section{Conclusions}

Cleavage embryo transfer should be performed first to reduce the cycle cancellation rate. If the number of retrieved oocytes is more than eight, then blastocyst transplantation can be considered after fully discussing the advantages and disadvantages of blastocyst culture with patients. Alternatively, cleavage embryo transfer can be performed first, and frozen-thawed blastocyst transfer can be performed next if cleavage embryo transfer is unsuccessful.

\section{Background}

Embryo transfer to the uterus during in vitro fertilisation treatment is an important procedure in assisted reproductive technology (ART). Currently, D3 cleavage embryo transfer is widely accepted by most centres. However, during the past 10 years, especially with improvements in embryo culture systems, an increasing number of centres have chosen blastocyst culture $(1,2)$. Many studies have confirmed that blastocyst transfer can result in higher clinical pregnancy rates and live birth rates than cleavage embryo transfer (3-5). This has led physicians to consider using D5/D6 blastocysts for transfer. With the improvements in freezing technology, some centres have advocated whole embryo freezing and suggested freeze-thawed blastocyst transfer $(6,7)$. However, a Cochrane review published in 2016 
concluded that there was no evidence of any difference in the cumulative pregnancy rates after fresh embryo transfer and frozen-thawed embryo transfer after one-oocyte retrieval (odds ratio [OR], 0.89; 95\% confidence interval [Cl], 0.64-1.22; very low-quality evidence) (5). More importantly, during the process of embryo self-selection, it is difficult for some embryos to form blastocysts. Therefore, if blastocyst transfer is planned, then the possibility of cycle cancellation for many patients is greatly increased $(3,4)$ because not all cycles are suitable for blastocyst culture, especially when patients have poor ovarian response and fewer oocytes (8). Therefore, the suitability of blastocyst transplantation for older patients has been questioned. Most previous studies have been based on the younger population, and the optimal embryo transfer scheme for older women is controversial (9-11). At present, many patients seeking ART treatment are in their late 30 s or early 40 s before they are diagnosed with infertility. Age is an important factor affecting the outcome of ART. With increasing age, the number and quality of oocytes decrease to varying degrees (12). Studies have shown that the copy number of mitochondrial DNA in the oocytes of older patients is significantly lower than that in the oocytes of younger patients (13). The incidence of aneuploidy in older patients is significantly higher than that in younger patients (14). Studies have shown that the cumulative live birth rate resulting from ART decreases significantly after age 35 years $(15,16)$. Therefore, the embryo transfer strategy for older women is different from that for younger women.

The time required to achieve live birth has become an important factor in ART. It is necessary to help patients achieve live birth efficiently within the shortest time while avoiding complications (such as hyperstimulation syndrome and multiple births) $(16,17)$. Studies have shown that the older the patient, the lower the pregnancy rate and live birth rate (18). Therefore, time is more important to and achieving live birth within a short time span is an urgent matter for older patients. This study aimed to compare the live birth rate and clinical pregnancy rate resulting from fresh cleavage embryo transfer (CET), frozenthawed cleavage embryo transfer (FCET), and frozen-thawed blastocyst transfer (FBT) cycles for patients older than 40 years, and to explore a more suitable transfer strategy for older patients to help them achieve live birth within the shortest time.

\section{Methods}

\section{Study population and design}

During this retrospective study, we analysed the fresh cycle data of older patients over the course of the past 10 years, analysed the embryonic development of the fresh cycle, and calculated the blastocyst formation rate. Clinical and neonatal outcomes of fresh CET, FCET, and FBT cycles for older patients over the course of the past 10 years were compared. Additionally, pregnancy outcomes of the transfer of two fresh cleaved embryos and the transfer of one frozen-thawed blastocyst were compared to provide a theoretical basis for deciding which embryo transfer strategy is best for older patients.

Data of patients older than 40 years who underwent ART treatment, including CET, FCET, and FBT, at the Assisted Reproductive Center of Peking Union Medical College Hospital from 1 January 2009 to 31 December 2019 were collected. Patients with no retrieved oocytes, donated eggs, or frozen eggs and 
patients who were lost to follow-up during this study were excluded. A total of 1233 CET cycles and 426 freeze-thaw cycles were included, including 396 FBT cycles and 30 FCET cycles. Patients who underwent FCET were further divided into two groups according to their age at the time of oocyte retrieval (younger than 40 years group and 40 years and older group). Finally, 280 FBT cycles and 26 FCET cycles were included. Figure 1 presents a flowchart of the inclusion of patients in this study. All patients signed a written informed consent form. This study was approved by the Ethics Committee of the Peking Union Medical College Hospital in China. All methods were performed in accordance with the relevant guidelines and regulations.

\section{Treatment protocol and embryo evaluation}

After pituitary suppression with gonadotropin-releasing hormone agonist and gonadotropin-releasing hormone antagonist, follicle-stimulating hormone and human menopausal gonadotropin were used for ovarian stimulation. When three follicles with diameters of 18 to $20 \mathrm{~mm}$ were observed, $250 \mu \mathrm{g}$ of human chorionic gonadotropin was injected to reach final maturation. Then, oocytes were obtained under transvaginal ultrasound guidance $38 \mathrm{~h}$ later. In vitro fertilisation or intracytoplasmic sperm injection was performed based on the semen quality. The zygotes and embryos were cultured in an incubator at $37^{\circ} \mathrm{C}$ and in an atmosphere containing $6 \% \mathrm{CO}_{2}, 5 \% \mathrm{O}_{2}$, and $89 \% \mathrm{~N}_{2}$. Embryos were evaluated on day 3 after fertilisation. We defined the high-quality cleavage stage embryos as those with seven or eight blastomeres on day 3 after fertilisation with uniform cell size, no multinucleation, and less than $20 \%$ fragmentation. The others were not high-quality embryos. One or two embryos with the best quality were selected for transplantation. All the embryo transfers were performed by the senior embryologists with more than 5 years of experience working in the embryo laboratory. If the embryos could not be transplanted because the women were at risk for ovarian hyperstimulation or other reasons on the day of transplantation, then the embryos were frozen or all embryos were cultured to the blastocyst stage. After informed consent was obtained from the patients, all remaining embryos were transferred to blastocyst culture media and continuously cultured to D5 or D6 to observe blastocyst formation in the same incubator. At our centre, the blastocysts were evaluated using the Gardner score (19), which is based on the degree of expansion of the blastocyst cavity, the quality of the inner cell mass, and trophoblast cells. We defined blastocysts with an inner cell mass and trophoblast mass of $A$ or $B$ (grades: $A A, A B, B A$, and $\mathrm{BB}$ ) as high-quality embryos; those with a grade of $\mathrm{BC}$ were defined as embryos that were not of high quality. Only blastocysts with an inner cell mass with grade A or grade B were selected for freezing. Grade $\mathrm{C}$ blastocysts were abandoned, but the quality of the trophoblast layer was not limiting.

\section{Vitrification and warming of blastocysts}

For vitrification, blastocysts were artificially shrunk before freezing. A mixture of $15 \%$ DMSO, $15 \%$ glycol, $0.65 \mathrm{~mol} / \mathrm{L}$ sucrose, and $10 \mathrm{mg} / \mathrm{ml}$ Ficoll was used as a cryoprotectant solution. To warm the vitrified blastocysts, 0.33-M, 0.2-M, and 0-M sucrose solutions were used as a cryoprotectant diluent, and all steps 
were performed at $37^{\circ} \mathrm{C}$. All vitrified and warmed blastocysts underwent laser-assisted hatching, and approximately $25 \%$ of the zona pellucida was separated from the cell mass. Blastocysts were transferred to the blastocyst culture medium prepared in advance and placed in an incubator at $37^{\circ} \mathrm{C}$ with $6 \% \mathrm{CO}_{2}$ and $5 \% \mathrm{O}_{2}$. Cleavage embryos were cultured for $16 \mathrm{~h}$, and blastocysts were cultured for $2 \mathrm{~h}$. Embryo survival was evaluated before transplantation.

Endometrial preparation was performed according to the needs of the patient using either the natural cycle method or the artificial cycle method. The natural cycle method was used for patients with a regular menstrual cycle and normal ovulation. Before ovulation, $2000 \mathrm{IU}$ human chorionic gonadotropin was injected and luteal support (intramuscular injection of progesterone $20-40 \mathrm{mg} / \mathrm{d}$ ) was provided after follicle rupture was detected by ultrasound. Embryo transplantation was performed on day 6 after ovulation. The artificial cycle method included oral estradiol valerate administered on day 2 to day 4 of menstruation. Different starting doses (2-18 $\mathrm{mg} /$ day) were selected according to the previous endometrial conditions of patients. The thickness of the endometrium was monitored using ultrasound. When the thickness of the endometrium reached $8 \mathrm{~mm}$, the blastocysts were thawed and transferred. After transplantation, luteal support was started using Crinone vaginal gel $8 \%$ or progesterone injected intramuscularly.

\section{Outcome measures}

Clinical pregnancy was determined by an ultrasound examination indicating at least one foetus with a heartbeat at 6 to 7 weeks of gestation. Spontaneous abortion was defined as an intrauterine pregnancy loss during the pregnancy. A live birth was defined as a viable infant born at more than 28 weeks of gestation after embryo transfer. The neonatal outcome was evaluated based on characteristics such as gestational age at delivery, preterm birth (before 37 weeks of gestation), low birth weight ( $<2500 \mathrm{~g}$ at birth), and high birth weight ( $\geq 4000 \mathrm{~g}$ at birth).

\section{Statistical analysis}

SPSS 22.0 software (SPSS, IBM, Armonk, NY, USA) was used for statistical analyses. The main outcome measure was the live birth rate. The secondary outcome measures were the clinical pregnancy rate, spontaneous abortion rate, and neonatal outcome. Depending on the data distribution, a comparison of the means of the two groups was performed using either Student's t-test or the Mann-Whitney U test. A one-way analysis of variance was used to compare the means among the groups. The measurement data were evaluated using the chi-square test or Fisher's exact test. A multivariate logistic regression analysis was performed. Statistical significance was considered when $\mathrm{P}<0.05$.

\section{Results}


A total of 1259 fresh oocyte retrieval cycles were collected, and 1233 cycles were included after excluding invalid cycles. The average age of the patients was $41.8 \pm 1.7$ years. The average number of retrieved eggs was $5.7 \pm 3.9$. The blastocyst formation rate was $23.5 \%$. The freezing cycle rate was $19.8 \%$. Furthermore, 210 fresh embryo transfer cycles were cancelled for various reasons; $41.4 \%$ of these were cancelled because of poor embryo quality. The number of fresh embryo transfer cycles was 1023, and the transfer cycle rate was $83.0 \%$. A total of 453 cycles had no remaining embryos after transplantation. A total of 570 cycles could be continued to blastocyst cultures (Table 1). 
Table 1

Fresh cycle data of patients older than 40 years

\begin{tabular}{|ll|}
\hline Items & Results \\
\hline Cycles (n) & 1252 \\
\hline Cycles with no oocytes (n) & 19 \\
\hline Effective cycles (n) & 1233 \\
\hline Age (y) & $41.8 \pm 1.7$ \\
\hline Infertile time (y) & $5.6 \pm 4.1$ \\
\hline BMI (kg/m²) (mean \pm SD) & $21.8 \pm 3.1$ \\
\hline Oocytes & $5.7 \pm 3.9(1-26)$ \\
\hline Fertilization rate (\%) & \\
\hline IVF (\%) & $3352 / 4536(73.9)$ \\
\hline ICSI (\%) & $1807 / 2053(88.0)$ \\
\hline High-quality embryo rate (\%) & $612 / 5142(11.9)$ \\
\hline Blastocyst formation rate (\%) & $704 / 2991(23.5)$ \\
\hline Freezing cycle rate (\%) & $244 / 1233(19.8)$ \\
\hline Transplantation rate (\%) & $1023 / 1233(83.0)$ \\
\hline Cycles after transplantation (n) & 499 \\
\hline Transplantation cancel rate (\%) & $210 / 1233(17.0)$ \\
\hline Poor-quality embryos (\%) & $87(41.4)$ \\
\hline OHSS (\%) & $18(8.6)$ \\
\hline Adenomyosis (\%) & $42(20)$ \\
\hline Other (\%) & $63(30)$ \\
\hline Ry & \\
\hline
\end{tabular}

BMI: body mass index; ICSI: intracytoplasmic sperm injection; IVF: in vitro fertilization, OHSS: ovarian hyperstimulation syndrome; SD: standard deviation.

There were no significant differences in the age, body mass index, duration of infertility, and type of infertility among the three groups. However, there were significant differences in the number of transferred embryos and the rate of transfer of high-quality embryos $(P<0.05)$. The number of embryos transferred in the FBT group was significantly lower than that in the CET and FCET groups; however, the high-quality embryo rate in the FBT group was significantly higher than that in the CET and FCET groups ( 23.3 vs. 26.7 vs. $46.0 ; \mathrm{P}<0.01$ ). Among the CET group, FCET group, and FBT group, the implantation 
rates ( 9.7 vs. 10 vs. $1.8 ; P<0.05)$, biochemical pregnancy rates ( 3.6 vs. 3.8 vs. $14.3 ; P<0.05$ ), clinical pregnancy rates $(17.5$ vs. 19.2 vs. $27.1 ; P<0.05)$, and live birth rates $(11.1$ vs. 15.4 vs. $16.8 ; P<0.05)$ were significantly different.

There were no significant differences in multiple pregnancy rates and spontaneous abortion rates among the three groups. The incidence of spontaneous disappearance of twins in the CET group was higher than that in the FBT group ( 40.9 vs. $14.3 \% ; P=0.20$ ). The twin birth rate in the CET group was lower than that in the FCET and FBT groups, although the difference was not significant ( 7.9 vs. $25.0 \%$ vs. $12.8 \%$; $=$ 0.37). There were no significant differences in gestational age, birth weight, and body length among the three groups. There was no significant difference in the incidence of premature birth and low birth weight among the three groups. There was one case of neonatal malformation in the FBT group. Although the sex ratios (male:female) of the FCET and FBT groups were higher than that of the CET group, there was no significant difference among the three groups (Table 2). 
Table 2

General conditions, clinical outcomes, and neonatal outcomes of the CET, FCET, and FBT groups

\begin{tabular}{|c|c|c|c|c|}
\hline Items & $\begin{array}{l}\text { CET group }(n= \\
1023)\end{array}$ & $\begin{array}{l}\text { FCET group ( } n \\
=26)\end{array}$ & $\begin{array}{l}\text { FBT group }(n= \\
280)\end{array}$ & $P$ \\
\hline Age (y) & $41.8 \pm 1.6$ & $42.7 \pm 2.1$ & $41.7 \pm 1.6$ & 0.02 \\
\hline $\begin{array}{l}\text { Time since infertility diagnosis } \\
\text { (y) }\end{array}$ & $5.6 \pm 4.2$ & $8.1 \pm 4.1$ & $6.7 \pm 4.5$ & 0.71 \\
\hline $\mathrm{BMI}\left(\mathrm{kg} / \mathrm{m}^{2}\right)($ mean $\pm \mathrm{SD})$ & $21.8 \pm 3.2$ & $21.7 \pm 3.2$ & $21.8 \pm 3.1$ & 0.61 \\
\hline Infertility types, n (\%) & & & & 0.25 \\
\hline Primary (\%) & $479(38.8)$ & $13(50)$ & $100(35.7)$ & \\
\hline Secondary (\%) & $754(61.2)$ & $13(50)$ & $180(64.2)$ & \\
\hline Transferred embryos (n) & $2.03 \pm 0.6$ & $2.3 \pm 0.5$ & $1.6 \pm 0.5$ & 0.03 \\
\hline Excellent embryos transferred (\%) & $483 / 2074(23.3)$ & $16 / 60(26.7)$ & 195/442 (46.0) & 0.00 \\
\hline Implantation (\%) & $201 / 2074(9.7)$ & $6 / 60(10)$ & 83/442 (18.8) & 0.00 \\
\hline Multiple births (\%) & $22 / 179(12.2)$ & $1 / 5(20)$ & $7 / 76(9.2)$ & 0.65 \\
\hline $\begin{array}{l}\text { Natural disappearance of one } \\
\text { twin }(\%)\end{array}$ & $9 / 22(40.9)$ & $0(0)$ & $1 / 7(14.3)$ & 0.20 \\
\hline Biochemical pregnancy (\%) & $37(33.6)$ & $1(3.8)$ & $40(14.3)$ & 0.00 \\
\hline Clinical pregnancy (\%) & $179(17.5)$ & $5(19.2)$ & $76(27.1)$ & 0.002 \\
\hline Spontaneous abortion (\%) & $65(36.3)$ & $1(20)$ & $28(36.8)$ & 0.75 \\
\hline Live birth (\%) & $114(11.1)$ & $4(15.4)$ & $47(16.8)$ & 0.04 \\
\hline \multicolumn{5}{|l|}{ Comparison of neonatal conditions } \\
\hline Deliveries (n) & 114 & 4 & 47 & \\
\hline Twin, $\mathrm{n}(\%)$ & $9(7.9)$ & $1(25.0)$ & $6(12.8)$ & 0.37 \\
\hline Gestational age $(\mathrm{d})$ & $269.4 \pm 12.3$ & $271.3 \pm 6.4$ & $268.1 \pm 16.0$ & 0.23 \\
\hline $\begin{array}{l}\text { Preterm birth (gestational age }< \\
37 \text { weeks) }\end{array}$ & $8(7.0)$ & 0 & $6(12.8)$ & 0.40 \\
\hline Birth weight (g) & $3079.3 \pm 555.6$ & $3134 \pm 541.8$ & $3113.7 \pm 641.1$ & 0.51 \\
\hline Low birth weight (\%) & $13(10.6)$ & 0 & $7(13.2)$ & 0.24 \\
\hline Low birth weight (\%) & $5(4.1)$ & 0 & $3(5.7)$ & 0.52 \\
\hline
\end{tabular}

CET group: fresh cleavage embryo transfer; FBT: frozen-thawed blastocyst embryo transfer; FCET group: frozen-thawed cleavage embryo transfer. $P<0.05$ indicates statistical significance. 


\begin{tabular}{|lllll|}
\hline Items & $\begin{array}{l}\text { CET group }(\mathbf{n}= \\
\text { 1023) }\end{array}$ & $\begin{array}{l}\text { FCET group }(\mathbf{n} \\
\mathbf{=} 26)\end{array}$ & $\begin{array}{l}\text { FBT group }(\mathrm{n}= \\
\mathbf{2 8 0})\end{array}$ & $\boldsymbol{P}$ \\
\hline Birth defects (\%) & 0 & 0 & $1(1.9)$ & 0.30 \\
\hline Sex (male/female) & $53 / 70$ & $4 / 1$ & $30 / 23$ & 0.065 \\
\hline $\begin{array}{l}\text { CET group: fresh cleavage embryo transfer; FBT: frozen-thawed blastocyst embryo transfer; FCET } \\
\text { group: frozen-thawed cleavage embryo transfer. } P<0.05 \text { indicates statistical significance. }\end{array}$ \\
\hline
\end{tabular}

The logistic regression analysis showed that the older the patient, the lower the live birth rate (OR, 0.776 ; $95 \% \mathrm{Cl}, 0.685-0.878$ ) (Table 3 ). The number of transferred embryos and the number of transferred highquality embryos were related to the live birth rate $(P=0.003$ and $P=0.008$, respectively). The live birth rate of two-embryo transplantation was 2.371-times that of one-embryo transplantation. Although the live birth rate of three-embryo transplantations was 1.862-times higher, the difference was not significant $(P=$ 0.06). The live birth rates of transplantations involving one high-quality embryo and two high-quality embryos were 1.792-times and 1.232-times higher than that of one-embryo and two-embryo transplantation, respectively. Compared with the CET group, the FCET group did not have an increased live birth rate $(P=0.348)$, but the FBT group had a significantly higher live birth rate $(O R, 1.883 ; 95 \% \mathrm{Cl}$, 1.932-4.535; $P=0.002)$. 
Table 3

Multiple logistic regression analysis of clinical pregnancy rates and live birth rates of older patients

\begin{tabular}{|c|c|c|c|c|}
\hline & \multicolumn{2}{|l|}{ Clinical pregnancy } & \multicolumn{2}{|l|}{ Live birth } \\
\hline & OR $(95 \% \mathrm{Cl})$ & $P$ & OR $(95 \% \mathrm{Cl})$ & $P$ \\
\hline Age & $0.79(0.72-0.88)$ & 0.000 & $0.78(0.69-0.8)$ & 0.000 \\
\hline Transferred embryos (n) & & 0.003 & & 0.003 \\
\hline 1 & Reference & & Reference & \\
\hline 2 & $1.71(1.16-2.51)$ & 0.007 & $2.37(1.43-3.94)$ & 0.001 \\
\hline 3 & $1.034(0.61-1.76)$ & 0.90 & $1.86(0.97-3.57)$ & 0.06 \\
\hline Excellent embryos transferred (n) & & 0.003 & & 0.008 \\
\hline 0 & Reference & & Reference & \\
\hline 1 & $1.68(1.22-2.30)$ & 0.001 & $1.79(1.24-2.59)$ & 0.002 \\
\hline 2 & $1.57(1.03-2.37)$ & 0.035 & $1.23(0.74-2.05)$ & 0.42 \\
\hline Groups & & 0.005 & & 0.008 \\
\hline CET & Reference & & Reference & \\
\hline FBT & $1.77(1.26-2.49)$ & 0.001 & $1.88(1.25-2.83)$ & 0.002 \\
\hline FCET & $1.33(0.48-3.68)$ & 0.58 & $1.70(0.56-5.18)$ & 0.35 \\
\hline $\begin{array}{l}\text { CET group: fresh cleavage embryo } \\
\text { embryo transfer; FCET group: froze } \\
\text { indicates statistical significance. }\end{array}$ & $\begin{array}{l}\text { sfer; } \text { Cl: confidence } \\
\text { awed cleavage em }\end{array}$ & $\begin{array}{l}\text { rval; FB } \\
\text { transfel }\end{array}$ & $\begin{array}{l}\text { frozen-thawed bla } \\
\text { R: odds ratio. } P<\end{array}$ & yst \\
\hline
\end{tabular}

When comparing the pregnancy outcomes of two-embryo transfers in the CET group and one-embryo transfers in the FBT group, we found that the high-quality embryo rate of the FBT group was significantly higher than that of the two-embryo transfers in the CET group, and that the biochemical pregnancy rate and implantation rate were significantly higher than those of the CET group (Table 4). However, the spontaneous abortion rate for one-embryo transfers in the FBT group was higher, and there was no difference in the clinical pregnancy rates and live birth rates of the CET and FBT groups. However, the live birth rate of the two-embryo transfers in the CET group was higher than that in the FBT group $(12.2 \%$ vs. $6.8 \% ; P=0.09)$. 
Table 4

Clinical outcomes of two-embryo transfers and oneembryo transfers in the CET and FBT groups

\begin{tabular}{|c|c|c|c|}
\hline Items & $\begin{array}{l}\text { Two-embryo transfers in the } \\
\text { CET group }(n=617)\end{array}$ & $\begin{array}{l}\text { Oneembryo transfers in the } \\
\text { FBT group }(n=118)\end{array}$ & $P$ \\
\hline $\begin{array}{l}\text { Excellent embryos } \\
\text { transferred (\%) }\end{array}$ & $333 / 1234(22.7)$ & $52 / 118(44.1)$ & 0.00 \\
\hline Age $(y)$ & 41.8 & 42.0 & 0.81 \\
\hline Implantation (\%) & $147 / 1234(11.9)$ & $26 / 118$ (22.0) & 0.02 \\
\hline $\begin{array}{l}\text { Biochemical } \\
\text { pregnancy (\%) }\end{array}$ & $28(4.5)$ & $13(11.0)$ & 0.005 \\
\hline $\begin{array}{l}\text { Clinical pregnancy } \\
(\%)\end{array}$ & $129(20.9)$ & $26(22.0)$ & 0.78 \\
\hline $\begin{array}{l}\text { Spontaneous } \\
\text { abortion (\%) }\end{array}$ & $52(40.3)$ & $17(65.4)$ & 0.019 \\
\hline Live birth (\%) & 75 (12.2) & $8(6.8)$ & 0.09 \\
\hline
\end{tabular}

\section{Discussion}

Because our centre did not perform fresh blastocyst transfers, we compared the pregnancy outcomes of CET, FCET, and FBT during this study. However, by reviewing the literature, we found that with improvements in embryo freezing technology, cryopreservation of embryos can now be performed more safely $(20,21)$. At our centre, beginning in 2005 , all blastocysts were cryopreserved by vitrification, and the survival rate after resuscitation reached $99 \%$ (22). Additionally, studies have shown that the pregnancy rate after frozen-thawed blastocyst transplantation is similar to or even better than that after fresh blastocyst transplantation $(23,24)$. Similar to fresh blastocyst transplantation, frozen-thawed blastocysts can lead to live births efficiently and safely (24). Therefore, the comparison can also provide a reference value for the development of transplantation strategies for older patients.

During our study, the outcomes of fresh embryo transfer and frozen-thawed embryo transfer for older patients were compared. The pregnancy outcomes of CET, FCET, and FBT were also compared. The clinical pregnancy rate and live birth rate of the FBT group were significantly higher than those of the CET and FCET groups, similar to the findings of previous studies $(4,5,25,26)$. According to a Cochrane Review in 2016, the live birth rate after fresh transfer was higher in the blastocyst transfer group than in the cleavage transfer group $(\mathrm{OR}, 1.48 ; 95 \% \mathrm{Cl}, 1.20-1.82 ; 13$ randomised, controlled trials of 1630 women), thus indicating that if the live birth rate of fresh cleaved embryo transfer is $29 \%$, then that of blastocyst transfer will increase to $32-42 \%$ (5). The reason for this may be that prolonging embryo culture from the cleavage stage to the blastocyst stage can improve embryo self-selection because only 
the embryos that can develop into blastocysts are selected, thereby resulting in better evaluations of the implantation potential of embryos (4). Blastocyst transfer is better able to simulate the natural implantation time of embryos and results in better receptivity by the endometrium. Studies have shown that the endometrium after ovarian stimulation undergoes histological changes and gene expression changes and has structural abnormalities (26). Therefore, frozen-thawed embryo transfer can prevent the possible abnormal effects of gonadotropin on the endometrium and improve the live birth rate. As a result, more centres have adopted whole embryo freezing strategies. Additionally, the number of highquality embryos transferred in the FBT group was significantly higher than that transferred in the other two groups during this study, and the live birth rate of the FBT group was still significantly higher than that of the CET and FCET groups after the multivariate regression analysis, which is different from some research conclusions $(9,27)$. Levi-Setti et al. included individuals younger than 39 years in their study and compared the clinical outcomes of fresh blastocyst and cleaved embryo transfer (9). They found that blastocyst transfer did not significantly improve the pregnancy rate and live birth rate, but that it did increase the twin pregnancy rate; however, this may have been related to the younger age and better embryo quality of the patients (9).

Regarding the multiple birth rates, this study suggested that there was no significant difference among the three groups, but that multiple birth rates were slightly higher in the CET and FCET groups, which may have been related to our transplantation strategy. Compared with the number of embryos recommended by the American Society for Reproductive Medicine in 2013, for women 38 to 40 years of age, the recommended numbers of cleavage stage embryos and blastocyst stage embryos were three or four and two or three, respectively. For patients 41 to 42 years of age, no more than five cleavage stage embryos or no more than three blastocysts should be transferred (28). The number of transferred embryos was relatively conservative. According to the relevant regulations of China, two or three blastomeres or two blastocysts were transplanted to older patients. Because of the increasing attention focused on multiple pregnancies, our strategy was to reduce the number of embryos transferred; however, our criteria were more restrictive than those proposed by the American Society for Reproductive Medicine in 2017 (29). After 2017, we began transferring two cleaved embryos and one or two blastocysts to patients older than 40 years. During the past 3 years, we have gradually moved to single-blastocyst transplantation. Using a multiple logistic regression analysis, we found that two-embryo transplantation can significantly improve the clinical pregnancy rate and live birth rate, but that three-embryo transplantation did not show significant improvements, which may have been the result of the poor embryo quality of older patients, especially in the cleavage stage. Embryo quality is often poor when three embryos are transferred. Increasing the number of transferred embryos cannot effectively improve the live birth rate. Additionally, this study suggested that although the numbers of transferred embryos in the CET and FCET groups were significantly higher than the number transferred in the FBT group, the live birth rate was significantly lower than that of the FBT group, thus demonstrating the advantages of blastocyst transplantation.

The neonatal outcomes after blastocyst transplantation have been controversial. Studies have found that there are some problematic neonatal outcomes of blastocyst transplantation. Blastocyst transplantation is believed to be more prone to premature birth than cleavage embryo transfer (30-32). A meta-analysis 
conducted by Maheshwari et al. found that compared with cleavage embryo transfer, the incidence of preterm birth before 37 weeks of gestation was higher in the blastocyst transfer group (risk ratio, 1.27; $95 \% \mathrm{Cl}, 1.22-1.31)(31)$, and that the blastocyst transfer group was more likely to have infants who were large for gestational age (30). Similarly, Zhou et al. found that for patients younger than 35 years, the birth weight of the blastocyst transplantation group was significantly higher than that of the cleavage embryo transplantation group, which may have been caused by the effects of epigenetic alteration on the birth weight of newborns during blastocyst culture (11). However, for older patients, there was no difference in birth weight between the two groups, which was consistent with our findings. We found no significant differences in neonatal outcomes among the three groups, which is consistent with the conclusions of other studies $(33,34)$. During one study, the neonatal outcomes of singleton birth after blastocyst transplantation and cleavage embryo transplantation were similar when a fresh transfer cycle was involved. Blastocyst transplantation did not affect preterm birth or birth weight, and it did not alter the probability of the newborns being healthy (34). A similar conclusion was reached during a study of the frozen-thaw cycle that aimed to determine whether the transfer of the frozen-thawed cleavage embryo or blastocyst affected the neonatal weight. Additionally, during this study, we found that although the sex ratios (male:female) in the FCET and FBT groups were higher than that in the CET group, there was no significant difference among them, similar to the findings of other studies $(9,27,34,35)$. Marconi et al. found that extended culture was associated with a marginal increase in the chance of male embryos (adjusted risk ratio, $1.04 ; 99.5 \% \mathrm{Cl}, 1.01-1.09$ ) (34). However, several studies have suggested that blastocyst transfer can increase the proportion of male embryos $(3,36,37)$. Because male embryos develop faster than female embryos and have better morphology, the faster-growing embryos are more likely to be male (38). Additionally, studies have shown that compared with female embryos developed during the same period, the morphological score of male embryos was better; for example, $72 \%$ of D 5 or D6 blastocysts were male embryos, whereas $60 \%$ of D3 blastocysts were female embryos (39). However, the specific mechanism of action is not clear and requires further study.

Although blastocyst transplantation is efficient and can lead to a high live birth rate, blastocyst culture has some disadvantages. With the in vitro culture, a considerable number of embryos have low blastocyst formation rates because of poor embryo quality or atresia, especially for patients with a poor prognosis $(4,5)$. Some studies have shown that the blastocyst formation rate decreases with age $(40)$. If such patients choose blastocyst transfer, then a large proportion of these patients may be left without available embryos. Compared with cleavage embryo transfer, the cycle cancellation rate will be greatly increased. During this study, the age of patients who underwent fresh cleaved embryo transfer ranged from 40 to 48.8 years, the number of fresh embryo transfer cycles was 1023 , the transfer rate was $83.0 \%$, the blastocyst formation rate was $23.5 \%$, and the freezing cycle rate was $19.8 \%$. A total of 453 cycles had no remaining embryos after transplantation. This means that if these patients do not undergo fresh transplantation and all of them have blastocyst culture, then more than half of them will not have the chance to undergo transplantation. Additionally, the outcomes of two-embryo transfers in the CET group and one-embryo transfers in the FBT group were compared. We found that although the high-quality embryo rate of the one-embryo transfers in the FBT group was significantly higher than that of the two- 
embryo transfers in the CET group, there was no significant difference in the pregnancy rates of these two groups. In contrast, the spontaneous abortion rate of the two-embryo transfers in the CET group was significantly higher than that of the two-embryo transfers in the CET group, and the live birth rate of the one-embryo transfers in the FBT group was slightly lower than that of the two-embryo transfers in the CET group, but the difference was not significant. For older patients, especially those with a small number of embryos, if they are allowed to extend to culture blastocysts, even if they can develop blastocysts, then their chances of achieving live birth may not be as high as that of patients with transferred cleavage embryos. Furthermore, some studies have suggested that some cleaved embryos cannot develop to the blastocyst stage in vitro; however, they may develop into viable embryos in vivo after D3 transplantation (41). Therefore, early cleavage embryo transfer may be more effective in some cases. For older patients with a poor prognosis, careful decisions should be made to ensure that embryos of the best quality are transferred as soon as possible during the treatment process. The optimisation of these processes will reduce the time to live birth by reducing the need for treatment cycles. A recent review of evidence-based medicine showed that the whole embryo cryopreservation strategy does not improve the cumulative live birth rate and continued pregnancy rate $\left(\mathrm{OR}, 1.08 ; 95 \% \mathrm{Cl}, 0.95-1.22 ; \mathrm{I}^{2}=0 \% ; 8\right.$ randomised, controlled trials; 4712 women; model-quality evidence), and the time to pregnancy was not determined according to the data of the included studies; however, they found that under the premise of similar live birth rates, the time to pregnancy with the fresh embryo transfer strategy seemed to be slightly less than that of the whole embryo freezing strategy (42) because frozen-thawed embryo transplantation involves a longer waiting time than fresh transplantation. For older women, time is valuable because they hope to become pregnant sooner and have a live birth. It is recommended that they should start the next cycle as soon as possible if pregnancy does not occur. Therefore, it is suggested that D3 CET should be performed instead of D5/D6 blastocyst transfer for older women with fewer oocytes retrieved to reduce the cancellation rate of the transfer cycle and make it easier for them to accept the risk of CET (4). Based on the data of this study, the blastocyst formation rate of the older patients was $23.5 \%$; however, our blastocysts were raised from the remaining embryos after two embryos were transferred. If all blastocysts were not transferred, then the blastocyst formation rate would be higher ( $>23.5 \%$ ), which means that approximately three or four cleaved embryos are required to obtain one blastocyst. The live birth rate resulting from one blastocyst seems to be slightly lower than that resulting from two cleaved embryos. To achieve a higher live birth rate, it is better to obtain two blastocysts; therefore, more than seven or eight cleaved embryos are required. For patients with more than seven or eight embryos after fertilisation, blastocyst transfer can be considered if they are willing to accept the risk. Alternatively, CET can be performed first, and then FBT can be performed if CET is not successful.

There were some limitations to this study. First, the number of patients who had FCET cycles was relatively low, which may have affected the comparisons of the neonatal outcomes. In the future, a multicentre study with a large sample size should be conducted to address this issue. Second, this study was retrospective, and the rate of excellent embryos transferred during FBT cycles was higher than that transferred during the CET cycles. In the future, studies involving blastocysts of balanced quality should be conducted to address this. This would enable us to analyse whether there is a difference between CET 
and FBT cycles. Finally, there was no long-term follow-up of the newborns. Because normal development at birth does not guarantee that issues will not occur in the future, a long-term follow-up study of the children should be conducted to investigate whether any abnormalities develop later.

\section{Conclusions}

In conclusion, the age of patients seeking ART is increasing (16); therefore, it is important to help patients achieve live births as soon as possible. Although the efficiency of blastocyst transfer is high, not every cycle is suitable. If the cycle cancellation rate is too high, then many patients will not benefit from ART. Therefore, we should consider the transfer strategy based on the quantity and quality of embryos after fertilisation. However, these results should be further confirmed by multicentre, randomised, controlled trials with large sample sizes.

\section{Abbreviations}

ART, assisted reproductive technology; CET, cleavage embryo transfer; $\mathrm{Cl}$, confidence interval; $\mathrm{FBT}$, frozenthawed blastocyst transfer; FCET, frozen-thawed cleavage embryo transfer; OR, odds ratio.

\section{Declarations}

\section{Ethics approval and consent to participate}

This study was approved by the Ethics Committee of the Peking Union Medical College Hospital in China (zs-1214). The ethics committees waived the requirement for informed consent because the retrospective nature of this study.

\section{Consent for publication}

Not applicable

\section{Availability of data and materials}

All data generated or analysed during this study are included in this published article.

\section{Competing interests}

The authors declare that they have no competing interests.

\section{Funding}

Peking Union Medical College Hospital sedimentation fund (grant number: ZC201904363).

\section{Authors' contributions}


$X W$ and ZYS contributed to the conception and design of the study. XW and YLX performed the literature search. JRZ and ZYS were involved in statistical analysis. XW, YLX, and QY contributed to the interpretation of the results. XW was responsible for manuscript drafting. All authors read and approved the final manuscript.

\section{Acknowledgements}

The authors thank Editage (www.editage.cn) for English language editing.

\section{References}

1. Kissin DM., Kulkarni AD, Mneimneh A, Warner L, Boulet SL, Crawford S, et al. Embryo transfer practices and multiple births resulting from assisted reproductive technology: an opportunity for prevention. Fertil Steril. 2015;103:954-61.

2. Papanikolaou EG, Kolibianakis EM, Tournaye $\mathrm{H}$, Venetis CA, Fatemi H, Tarlatzis B, et al. Live birth rates after transfer of equal number of blastocysts or cleavage-stage embryos in IVF. A systematic review and meta-analysis. Hum Reprod. 2008;23:91-9.

3. Practice Committee of the American Society for Reproductive Medicine, Practice Committee of the Society for Assisted Reproductive Technology. Blastocyst culture and transfer in clinically assisted reproduction: a committee opinion. Fertil Steril. 2018;110:1246-52.

4. Glujovsky D, Farquhar C. Cleavage-stage or blastocyst transfer: what are the benefits and harms. Fertil Steril. 2016;106:244-50.

5. Glujovsky D, Farquhar C, Quinteiro RAM, Alvarez SCR, Blake D. Cleavage stage versus blastocyst stage embryo transfer in assisted reproductive technology. Cochrane Database Syst Rev.

2016:CD002118. doi: 10.1002/14651858.CD002118.pub5

6. Bosdou JK., Venetis CA, Tarlatzis BC, Grimbizis GF, Kolibianakis EM. Higher probability of live-birth in high, but not normal, responders after first frozen-embryo transfer in a freeze-only cycle strategy compared to fresh-embryo transfer: a meta-analysis. Hum Reprod. 2019;34:491-505.

7. Zhu Q, Chen Q, Wang L, Lu X, Lyu Q, Wang Y, et al. Live birth rates in the first complete IVF cycle among 20687 women using a freeze-all strategy. Hum Reprod. 2018;33:924-9.

8. Sfontouris IA, Martins WP, Nastri CO, Viana IG, Navarro PA, Raine-Fenning N, et al. Blastocyst culture using single versus sequential media in clinical IVF: a systematic review and meta-analysis of randomized controlled trials. J Assist Reprod Genet. 2016;33:1261-72.

9. Levi-Setti PE, Cirillo F, Smeraldi A, Morenghi E, Mulazzani GE, Albani E. No advantage of fresh blastocyst versus cleavage stage embryo transfer in women under the age of 39: a randomized controlled study. J Assist Reprod Genet. 2018;35:457-65. 
10. Liu J, Zheng J, Lei YL, Wen XF. Effects of endometrial preparations and transferred embryo types on pregnancy outcome from patients with advanced maternal age. Syst Biol Reprod Med. 2019;65:181-6.

11. Zhou QW, Jing S, Xu L, Guo H, Lu CF, Gong F, et al. Clinical and neonatal outcomes of patients of different ages following transfer of thawed cleavage embryos and blastocysts cultured from thawed cleavage-stage embryos. PLoS One. 2018;13:e0207340. doi: 10.1371/journal.pone.0207340

12. Broekmans FJ, Knauff EA, te Velde ER, Macklon NS, Fauser BC. Female reproductive ageing: current knowledge and future trends. Trends Endocrinol Metab. 2007;18:58-65.

13. Murakoshi Y, Sueoka K, Takahashi K, Sato S, Sakurai T, Tajima H, et al. Embryo developmental capability and pregnancy outcome are related to the mitochondrial DNA copy number and ooplasmic volume. J Assist Reprod Genet 2013;30:1367-75.

14. Minasi MG, Colasante A, Riccio T, Ruberti A, Casciani V, Scarselli F, et al. Correlation between aneuploidy, standard morphology evaluation and morphokinetic development in 1730 biopsied blastocysts: a consecutive case series study. Hum Reprod. 2016;31:2245-54.

15. American College of Obstetricians and Gynecologists Committee on Gynecologic Practice and Practice Committee. Female age-related fertility decline. Committee Opinion No. 589. Fertil Steril. 2014;101:633-4.

16. Coticchio G, Behr B, Campbell A, Meseguer M, Morbeck DE, Pisaturo V, et al. Fertility technologies and how to optimize laboratory performance to support the shortening of time to birth of a healthy singleton: a Delphi consensus. J Assist Reprod Genet. 2021;38:1021-43. doi: 10.1007/s10815-021-020775

17. Bosch E, Bulletti C, Copperman AB, Fanchin R, Yarali H, Petta CA, et al. How time to healthy singleton delivery could affect decision-making during infertility treatment: a Delphi consensus. Reprod Biomed Online. 2019;38:118-30.

18. Bortoletto P, Willson S, Romanski PA, Davis OK, Rosenwaks Z. Reproductive outcomes of women aged 40 and older undergoing IVF with donor sperm. Hum Reprod. 2021;36:229-35.

19. Gardner DK, Lane M, Stevens J, Schlenker T, Schoolcraft WB. Blastocyst score affects implantation and pregnancy outcome: towards a single blastocyst transfer. Fertil Steril. 2000;73:1155-8.

20. Rienzi L, Gracia C, Maggiulli R, LaBarbera AR, Kaser DJ, Ubaldi FM, et al. Oocyte, embryo and blastocyst cryopreservation in ART: systematic review and meta-analysis comparing slow-freezing versus vitrification to produce evidence for the development of global guidance. Hum Reprod Update. 2017;23:139-55.

21. Wong KM, Mastenbroek S, Repping, S. Cryopreservation of human embryos and its contribution to in vitro fertilization success rates. Fertil Steril. 2014;102:19-26. 
22. Wang X, Zhen J, Sun Z, Yu Q, Deng C, Zhou Y, et al. Effects of fifth day (D5) or sixth day (D6) frozen-thawed blastocysts on neonatal outcomes. Zygote. 2016;24:684-91.

23. Coates A, Kung A, Mounts E, Hesla J, Bankowski B, Barbieri E, et al. Optimal euploid embryo transfer strategy, fresh versus frozen, after preimplantation genetic screening with next generation sequencing: a randomized controlled trial. Fertil Steril. 2017;107:723-30.e3.

24. Wei D, Liu JY, Sun Y, Shi Y, Zhang B, Liu JQ, et al. Frozen versus fresh single blastocyst transfer in ovulatory women: a multicentre, randomised controlled trial. Lancet. 2019;393:1310-8.

25. Papanikolaou EG, Camus M, Kolibianakis EM, Van Landuyt L, Van Steirteghem A, Devroey P. In vitro fertilization with single blastocyst-stage versus single cleavage-stage embryos. N Engl J Med. 2006;354:1139-46.

26. Robinson RD. Success rates and pregnancy outcomes in thawed embryos transferred after extended culture: cryopreserved embryos versus cleavage stage cryopreserved embryos. Fertil Steril. 2018;110:59-60.

27. Li W, Xue X, Zhao W, Ren A, Zhuo W, Shi J. Blastocyst transfer is not associated with increased unfavorable obstetric and perinatal outcomes compared with cleavage-stage embryo transfer. Gynecol Endocrinol. 2017;33:857-60.

28. Practice Committee of the American Society for Reproductive Medicine, Practice Committee of the Society for Assisted Reproductive Technology. Criteria for number of embryos to transfer: a committee opinion. Fertil Steril. 2013;99:44-6.

29. Practice Committee of the American Society for Reproductive Medicine, Practice Committee of the Society for Assisted Reproductive Technology. Guidance on the limits to the number of embryos to transfer: a committee opinion. Fertil Steril. 2017;107:901-3.

30. Dar S, Lazer T, Shah PS, Librach CL. Neonatal outcomes among singleton births after blastocyst versus cleavage stage embryo transfer: a systematic review and meta-analysis. Hum Reprod Update. 2014;20:439-48.

31. Maheshwari A, Kalampokas T, Davidson J, Bhattacharya S. Obstetric and perinatal outcomes in singleton pregnancies resulting from the transfer of blastocyst-stage versus cleavage-stage embryos generated through in vitro fertilization treatment: a systematic review and meta-analysis. Fertil Steril. 2013;100:1615-21.

32. Wang X, Du M, Guan Y, Wang B, Zhang J, Liu Z. Comparative neonatal outcomes in singleton births from blastocyst transfers or cleavage-stage embryo transfers: a systematic review and metaanalysis. Reprod Biol Endocrinol. 2017;15:1-2. 
33. De Vos A, Dos SS, Tournaye H, Verheyen G. Birthweight of singletons born after blastocyst-stage or cleavage-stage transfer: analysis of a data set from three randomized controlled trials. $\mathrm{J}$ Assist Reprod Genet. 2020;37:127-32.

34. Marconi N, Raja EA, Bhattacharya S, Maheshwari, A. Perinatal outcomes in singleton live births after fresh blastocyst-stage embryo transfer: a retrospective analysis of 67147 IVF/ICSI cycles. Hum Reprod. 2019;34:1716-25.

35. Fang J, Zhu L, Li D, Xu Z, Yan G, Sun H, et al. Effect of embryo and blastocyst transfer on the birthweight of live-born singletons from FET cycles. J Assist Reprod Genet. 2018;35:1905-10.

36. Luna M, Duke M, Copperman A, Grunfeld L, Sandler B, Barritt J. Blastocyst embryo transfer is associated with a sex-ratio imbalance in favor of male offspring. Fertil Steril. 2007;87:519-23.

37. Maalouf WE, Mincheva MN, Campbell BK, Hardy IC. Effects of assisted reproductive technologies on human sex ratio at birth. Fertil Steril. 2014;101:1321-5.

38. Kawase $\mathrm{Y}$, Tachibe T, Kamada N, Jishage KI, Watanabe H, Suzuki H. et al. Male advantage observed for in vitro fertilization mouse embryos exhibiting early cleavage. Reprod Med Biol. 2021;20:837.

39. Alfarawati S, Fragouli E, Colls P, Stevens J, Gutiérrez-Mateo C, Schoolcraft WB, et al. The relationship between blastocyst morphology, chromosomal abnormality, and embryo gender. Fertil Steril. 2011;95:520-4.

40. Alhelou Y, Adenan NA, Ali J. Embryo culture conditions are significantly improved during uninterrupted incubation: a randomized controlled trial. Reprod Biol. 2018;18:40-5.

41. Gleicher N, Kushnir VA, Barad, D.H. Is it time for a paradigm shift in understanding embryo selection. Reprod Biol Endocrinol. 2015;13:3.

42. Zaat $\mathrm{T}$, Zagers $\mathrm{M}, \mathrm{Mol} \mathrm{F}$, Goddijn $\mathrm{M}$, Wely $\mathrm{M}$, Mastenbroek $\mathrm{S}$. Fresh versus frozen embryo transfers in assisted reproduction. Cochrane Database Sys Rev. 2021;2:CD011184. doi:

10.1002/14651858.CD011184.pub3

\section{Figures}




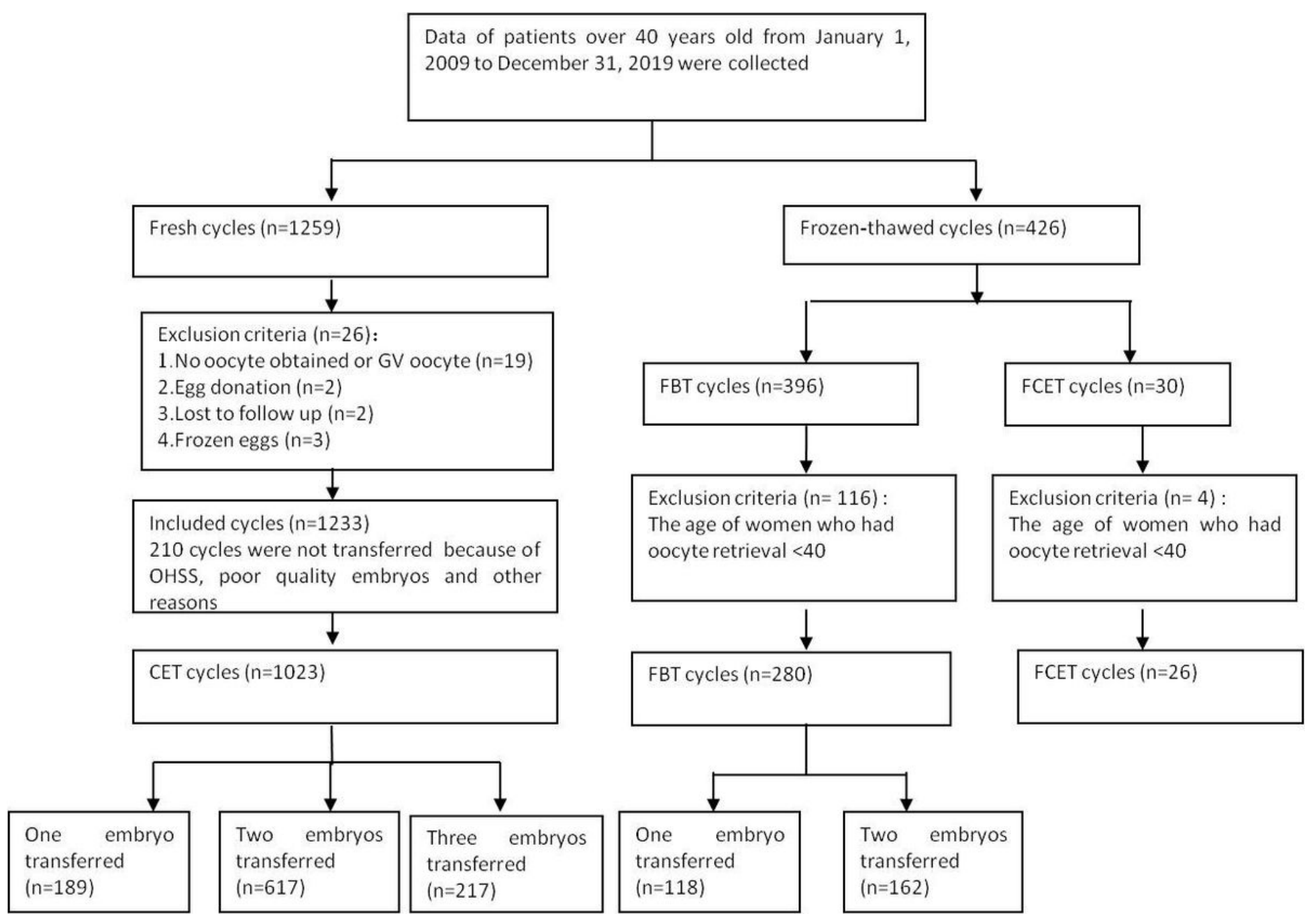

Figure 1

Flow chart of the patient inclusion process during this study. 